\title{
Comparação entre Dois Sistemas de Informação em Saúde sobre Hipertensão Arterial Sistêmica (HAS): Considerações sobre uma Experiência
}

\section{Comparison between Two Information Systems in Health about Systemic Arterial Hypertension (SAH): Considerations about an Experience}

\section{RESUMO}

Introdução: Objetivou comparar e compreender a lógica existente na produção, fluxo e análise dos dados que alimentam o Sistema de Cadastramento e Acompanhamento de Hipertensos e Diabéticos e o formato de Coleta de dados Simplificada do e-SUS Atenção Básica e identificar os desafios encontrados na mudança de um sistema para o outro no que diz respeito ao usuário com hipertensão. Metodologia: Trata-se de um estudo de caso que foi realizado com base na consulta de artigos científicos, documentos e manuais técnicos, relatórios de oficinas de trabalho, opiniões de trabalhadores da APS e vivências durante o processo de concepção e implantação dos dois sistemas. Resultados: Os Sistemas estudados apresentam diferenças entre as informações contidas nos mesmos e que existem divergências na produção, fluxo e análise dos dados entre os dois sistemas, entretanto, o que se percebe é que no HIPERDIA, os dados produzidos na Unidade são referentes aos usuários hipertensos cadastrados e acompanhados mensalmente e que os dados provenientes do e-SUS Atenção Básica formato de Coleta de dados Simplificada são referentes ao curso natural do atendimento. Conclusão: O presente estudo possibilitou a discussão dos dados referentes à hipertensão arterial a partir de duas bases de dados investigadas para Atenção Básica. À escassa literatura cientifica que discuta e reflita sobre a produção, fluxo e análise dos dados do formato de Coleta de dados Simplificada do e-SUS Atenção Básica como um todo, apresenta-se como uma limitação e, portanto, se faz necessário a realização de novas pesquisas sobre a temática.

DESCRITORES: Sistema de Informação; Hipertensão; Atenção Primária à Saúde; Estudos de Casos.

\begin{abstract}
Introduction: This study aimed to compare and understand the existing logic in the production, flow and analysis of data that feed the System of Registration and Monitoring of Hypertensive Diabetics and the simplified data collection format e-NHS Primary Care, and identify the challenges encountered in changing from one system to the other with regards to the user with hypertension. Methodology: This is a case study based on scientific articles query, documents and technical manuals, workshops reports, opinions of employees of APS and experiences during the process of design and implementation of the two system. Results: The studied systems differ from the information contained in them and there are differences in the production, flow and analysis of data between the two systems, however, it was noticed that in HIPERDIA, the data produced in the unit refer to hypertensive registered and monitored monthly users and the data from the e-SUS Primary Streamlined data collection format refer to the natural course of care. Conclusion: This study enabled the discussion of data on hypertension from two databases investigated for Primary Care. In the scarce scientific literature to discuss and reflect on the production, flow and analysis of simplified data collection format data from e-NHS Primary Care as a whole, it appears as a limitation and therefore it is necessary to carry out new research on the subject.
\end{abstract}

DESCRIPTORS: Information Systems; Hypertension; Primary Health Care; Case Studies.

1- Fisioterapeuta Doutorando, Pós-Graduação em Modelos de Decisão e Saúde, Universidade Federal da Paraíba. João Pessoa (PB), Brasil.

2- Estatístico. Pós-Doutor em Saúde Pública. Professor Titular da Universidade Federal da Paraíba (UFPB). João Pessoa (PB), Brasil.

3- Fisioterapeuta. Doutora em Educação. Professora Associada da Universidade Federal da Paraíba (UFPB). João Pessoa (PB), Brasil.

4- Enfermeira. Especialista em Saúde da Família. Secretaria Municipal de Pedras de Fogo. João Pessoa (PB), Brasil. 
A Hipertensão Arterial Sistêmica (HAS) é uma doença crônica de caráter multifatorial, assintomática conceituada como uma enfermidade que se caracteriza pelos níveis elevados e sustentados da Pressão Arterial (PA). Ela é considerada o principal fator de risco para as doenças cardiovasculares no Brasil e nos países desenvolvidos ${ }^{1}$, e faz parte de uma classe de doenças crônicas não transmissíveis (DCNT), responsáveis pelas maiores taxas de morbidade e mortalidade no país, ocasionando expressivas taxas de internação e custos elevados devido às intercorrências associada à mesma ${ }^{2,3}$.

No Brasil, a proporção de indivíduos com HAS representa uma prevalência entre 10 a $42 \%$ da população brasileira, dependendo da região, do critério de diagnóstico adotado e do subgrupo populacional, afetando tanto jovens quanto idosos ${ }^{4,5}$. No Nordeste, foi observada uma prevalência de HAS entre 7,2\% a 40,3\% ${ }^{6}$. Em João Pessoa-PB, a prevalência diagnosticada em 2011 foi de $25,4 \%$, sendo $28,7 \%$ entre as mulheres e $21,4 \%$ entre os homens ${ }^{7}$.

Devido a sua alta prevalência, a HAS vem sendo reconhecida pelo Ministério da Saúde (MS) como importante problema de saúde pública, onde o mesmo estabeleceu diretrizes para o cadastramento, acompanhamento e tratamento destes indivíduos ${ }^{1}$.

Essas diretrizes buscam desenvolver ações na Atenção Primária à Saúde (APS) a partir da comunidade, a fim de que o hipertenso possa ter uma assistência adequada, cadastramento e com acompanhamento constante e, assim, diminuir as complicações que a doença pode desenvolver ${ }^{8}$.

A consolidação dessas ações se deu por meio do cadastramento informatizado, realizado a partir da iniciativa do MS e intitulado SIS-HIPERDIA (Sistema de Cadastramento e Acompanhamento de Hipertensos e Diabéticos) que foi desenvolvido em 2001 com o propósito de reorganizar os serviços, para oferecer atenção qualificada e contínua aos portadores de HAS e/ou Diabetes Mellitus (DM) ${ }^{9}$.

O SIS-HIPERDIA permite cadastrar e acompanhar os portadores de HAS e DM, captados em toda a rede de APS do Sistema Único de Saúde (SUS). A partir dos cadastros locais, esse sistema pode gerar informações para os gerentes locais, gestores das secretarias municipais, estaduais e MS, além de ser uma ferramenta útil para profissionais da rede básica no enfrentamento destas doenças ${ }^{9}$.

Os sistemas, as tecnologias de informação e os registros computadorizados no SUS permitem que a equipe cuidadora acesse informações e dados clínicos do paciente de maneira ágil e oportuna, contribuindo para o melhor gerenciamento do cuidado e o monitoramento dos resultados.

Pensando na disponibilidade de novas tecnologias e de sistemas de informação como ferramentas para monitorar o cuidado, o Ministério da Saúde, a partir do Projeto de Reestruturação do Sistema de Informação da Atenção Básica (AB), em março de 2011 e consolidado por meio da publicação da Portaria GM/MS n. 1412, de 10 de julho de $2013^{10}$, lança o novo Sistema de Informação em Saúde da Atenção Básica (SISAB) e implanta o sistema informatizado único, denominado e-SUS AB.

O sistema e-SUS AB é composto pelo Prontuário Eletrônico do Cidadão (PEC) e pela Coleta de Dados Simplificada (CDS) cuja escolha pelo uso do CDS ou do PEC dependerá do cenário de implantação do e-SUS AB no município. Este sistema foi proposto para unificar o SISHIPERDIA e outros sistemas de informações em saúde, no prazo de um ano, consolidando as informações de cada indivíduo, entendendo que a qualificação da gestão da informação é fundamental para o aumento da qualidade do cuidado em saúde à população como o todo ${ }^{10}$.

Com esta nova proposta surgem questionamentos acerca do acesso às informações contidas no sistema anterior e a permuta de dados para o sistema atual, bem como as potencialidades de uso das informações sobre o usuário hipertenso cadastrado na $A B$.

Em 200911 Paes construiu uma coorte de hipertensos adultos cadastrados na $A B$ em municípios da Paraíba dando sequência nos anos $2010^{12}$ e $2011^{13}$. Durante o período da coorte ocorreu à transição do sistema SIS/HIPERDIA para o sistema e-SUS AB, ou seja, em 2011. Ao retomar esta coorte em 2015, o autor se defrontou com o problema de não mais poder fazer comparações dos perfis sociodemográficos, medições antropométricas e da pressão arterial, entre outros, dos usuários hipertensos, uma vez que os dados individuais não estão mais disponibilizados pelo sistema atual de captação destes dados na AB em João Pessoa. O comprometimento de estudos comparativos destes usuários para uma situação local levanta um problema que se estende a qualquer estudo com pretensões de conhecer as características individuais dos hipertensos de todo país.

Deste modo, faz-se necessário aprofundar 
e analisar as mudanças ocorridas a partir da implantação do novo sistema de informações e refletir acerca da repercussão dessas mudanças sobre o programa de controle da hipertensão na $A B$ sob diversos aspectos dimensionais. Essa análise poderá subsidiar outros estudos bem como auxiliar os órgãos de saúde, serviços públicos, profissionais e gestores para as tomadas de decisão.

Com base no exposto, este estudo teve como objetivo compreender a lógica existente na produção, fluxo e análise dos dados que alimentam o SIS/HIPERDIA e o formato de Coleta de Dados Simplificada (CDS) do e-SUS AB e, a partir daí, identificar os desafios encontrados na mudança de sistema de informação no que diz respeito ao usuário com hipertensão arterial na $A B$.

\section{METODOLOGIA}

Trata-se de um estudo de caso, para o qual foram traçadas estratégias metodológicas visando compreender a produção, fluxo e análise dos dados que alimentam o formato CDS do e-SUS e SIS/HIPERDIA desde o percurso do usuário ao acesso a APS, o seu cadastro como hipertenso, a produção da informação sobre Hipertensão Arterial na Unidade Básica de Saúde (UBS) até a alimentação do banco de dados no MS. Para tanto, deu-se destaque à comparação entre os sistemas enfocando o Município de João Pessoa-PB.

O presente trabalho foi realizado com base na consulta de artigos científicos, documentos e manuais técnicos, relatórios de oficinas de trabalho, opiniões de usuários e trabalhadores da APS e vivências da enfermeira da APS e Diretor Técnico do Distrito Sanitário $V$ do Município de João Pessoa-PB, durante o processo de concepção e implantação das versões do formato CDS do e-SUS AB e da utilização do HIPERDIA na APS.

O olhar crítico dos autores sobre o seu processo do trabalho e análise dos resultados das pesquisas anteriormente citadas, reflete-se no artigo aqui apresentado, que retrata a sua própria experiência da continuidade da coorte de hipertensos no município de João Pessoa-PB e na análise da comparação dos sistemas de informação em saúde que possa acolher as novas demandas institucionais definidas pelas normas reguladoras do SUS.

\section{RESULTADOS E DISCUSSÃO}

Os Sistemas de Informação HIPERDIA e do formato CDS do e-SUS foram criados com o intuito de acompanhar a produção de dados para assegurar avaliações da situação de saúde dos hipertensos e de toda a população, respectivamente e, assim, servirem como base para o planejamento do nível local como um instrumento para as práticas de atenção e de gestão.

Com a reformulação da Política Nacional da Atenção Básica (PNAB), aprovada pela Portaria MS/GM n. 2.488, de 21 de outubro de $2011^{14}$, ocorreu à revisão das diretrizes e normas para a Atenção Básica, ressaltando o exercício de práticas de cuidado e a gestão desenvolvida pelas equipes, a continuidade e a integralidade do cuidado, além do papel central da $A B$ nas Redes de Atenção à Saúde.

Diante das novas diretrizes apontadas pela PNAB, o Departamento de Atenção Básica do Ministério da Saúde (DAB/MS) elaborou a estratégia e-SUS Atenção Básica (e-SUS AB) para reestruturar o Sistema de Informação da Atenção Básica. Para isso, observa-se como fundamental o incremento da gestão da informação, seja melhorando as condições de infraestrutura e o processo de trabalho, seja demandando ferramentas e sistemas de informação.

O sistema de software e-SUS AB é público e auxilia no apoio à gestão do processo de trabalho e ao cuidado à saúde na Atenção Básica, que pode ser utilizado nos formatos de Coleta de Dados Simplificada (CDS) e Prontuário Eletrônico do Cidadão (PEC). A Coleta de Dados Simplificada (CDS) consiste no uso de fichas mais simples do que as usadas atualmente e que posteriormente deverão ser digitadas no sistema de software e-SUS AB CDS.

Ele é composto por 10 fichas para o registro de informações. São elas: cadastro domiciliar, cadastro individual, ficha de atendimento individual, ficha de atendimento odontológico individual, Avaliação da Elegibilidade e Admissão, Atendimento Domiciliar, Ficha de Visita Domiciliar, Ficha de Procedimentos e Ficha de Atividade Coletiva como evidenciado na Figura 1 a seguir.

O atual sistema foi desenvolvido para atender às necessidades da atenção básica, lotadas em unidades básicas de saúde, com baixa estrutura de informatização e para a gestão do cuidado em saúde. Pode ser utilizado por profissionais de todas as equipes de $A B$, pelas 

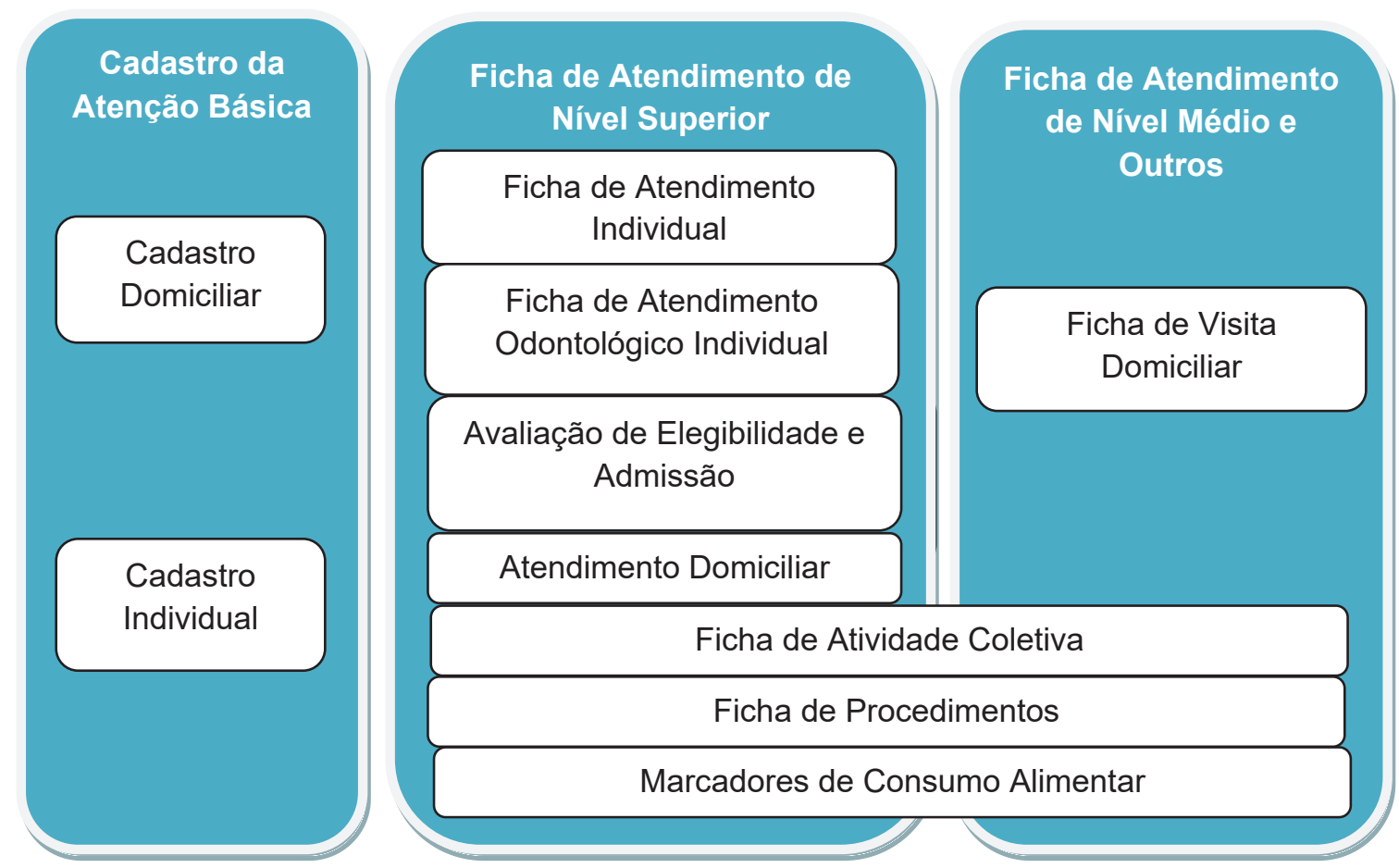

Fonte: Manual de Preenchimento das Fichas do Sistema com CDS, $2015^{15}$.

equipes dos Núcleos de Apoio à Saúde da Família (NASF), do Consultório na Rua (CnR), de Atenção à Saúde Prisional e da Atenção Domiciliar $(A D)$, além dos profissionais que realizam ações no âmbito de programas como o Saúde na Escola (PSE) e a Academia da Saúde ${ }^{15}$.

As informações referentes à hipertensão arterial são registradas nas fichas de cadastro individual, Ficha de Atendimento individual e Ficha de visita domiciliar, mas com informações que permitem a entrada ou não dos dados orientada pelo curso natural do atendimento e não focada na situação-problema de saúde referente à Hipertensão Arterial como no HIPERDIA.

Já o sistema com Prontuário Eletrônico do Cidadão (PEC) consiste na informatização de todos os dados dos pacientes que são atendidos na $A B$ e foi formulado para atender às equipes de $A B$ lotadas em Unidades Básicas de Saúde parcialmente ou totalmente informatizadas ${ }^{15}$.

As diferenças entre as informações contidas no HIPERDIA e formato CDS do e-SUS podem ser observadas nos quadros I e II.

O e-SUS apresenta-se como um sistema único e simplificado e as fichas de utilização do formato CDS acima descritas incluem algumas variáveis da ficha de cadastro do HIPERDIA. Entretanto, no formato CDS do e-SUS estão contempladas outras variáveis que não estavam no HIPERDIA.

A maioria das informações referentes à identificação do usuário presentes no HIPERDIA está contemplada nas fichas do formato CDS do e-SUS com exceção das variáveis: situação conjugal, nome do pai, data de naturalização e número da portaria. Já as variáveis, nome da Unidade, país de origem e escolaridade foram modificadas por outras, mas com significados parecidos.

No que diz respeito às informações referentes aos documentos gerais e obrigatórios presentes na ficha cadastral do HIPERDIA apenas o PIS/PASEP está contemplado na Ficha de Cadastro Individual do formato CDS do e-SUS. Em relação às variáveis de endereço do usuário contidas no HIPERDIA, todas estão contempladas na Ficha de cadastro domiciliar do formato CDS do e-SUS. 
Quadro I - Comparação das informações de Identificação do Usuário contidas no HIPERDIA e formato CDS do e-SUS.

\begin{tabular}{|c|c|}
\hline VARIÁVEIS DO HIPERDIA & VARIÁVEIS DO HIPERDIA NO e-SUS \\
\hline Nome da Unidade de Saúde & $\begin{array}{l}\text { Substituída por Código CNES da Unidade em todas as } \\
\text { fichas }\end{array}$ \\
\hline Número de Prontuário & Contemplada na Ficha de Cadastro Domiciliar \\
\hline Nome & Contemplada na Ficha de Cadastro Individual \\
\hline Data de Nascimento & Contemplada na Ficha de Cadastro Individual \\
\hline Sexo & Contemplada na Ficha de Cadastro Individual \\
\hline Nome da Mãe & Contemplada na Ficha de Cadastro Individual \\
\hline Nome do Pai & Não contemplada nas fichas do e-SUS \\
\hline Raça/Cor & Contemplada na Ficha de Cadastro Individual \\
\hline Escolaridade & $\begin{array}{l}\text { Contemplada na Ficha de Cadastro Individual, entretanto } \\
\text { com acréscimo das informações Creche, Pré-escola, } \\
\text { Classe alfabetizada, Ensino fundamental modalidades de } \\
1^{\text {a }} \text { a } 4^{a} \text { série, de } 5^{\mathrm{a}} \text { a } 8^{\mathrm{a}} \text { série, especial, EJA - séries } \\
\text { iniciais, EJA - séries finais, Ensino médio especial, Ensino } \\
\text { médio EJA, alfabetização para adultos (Mobral) }\end{array}$ \\
\hline Nacionalidade & Contemplada na Ficha de Cadastro Individual \\
\hline País de Origem & Substituída por país de Nascimento \\
\hline Data da Naturalização & Não contemplada nas fichas do e-SUS \\
\hline $\mathrm{N}^{0}$ da portaria & Não contemplada nas fichas do e-SUS \\
\hline UF Município de Nascimento & Contemplada na Ficha de Cadastro Individual \\
\hline Situação Familiar Conjugal & Não contemplada nas fichas do e-SUS \\
\hline $\mathrm{N}^{0}$ Cartão do SUS & Contemplada na Ficha de Cadastro Individual \\
\hline
\end{tabular}

Às informações referentes aos dados clínicos do paciente, às variáveis como Cintura, Amputação por Diabetes, Antecedentes Familiares cardiovasculares, sedentarismo, Pé Diabético não está contempladas nas fichas do formato CDS do e-SUS, enquanto que as demais informações detalhadas na tabela acima estão contempladas na Ficha de Cadastro Individual. Nenhuma variável referente ao tratamento seja ele medicamentoso ou não, contemplado na ficha do HIPERDIA está inserida na ficha do formato CDS do e-SUS.

O desafio estava lançado, uma vez que no prazo de um ano após a publicação da Portaria GM/MS n ${ }^{\circ}$. 1412, de 10 de julho de $2013^{10}$, as informações de cada indivíduo deveriam ser unificadas em um sistema apenas (e-SUS AB), suspendendo a alimentação e utilização dos sistemas anteriores. Para tanto, existiria quatro cenários possíveis de implantação do e-SUS dependendo de cada região e cidade brasileira. O primeiro seria o CDS-SMS onde o preenchimento das fichas do CDS aconteceria nas unidades básicas de saúde e a digitação das fichas em computadores centralizados na Secretaria Municipal de Saúde; o segundo seria o CDSUBS cujo preenchimento e digitação das fichas do CDS seriam nas unidades básicas de saúde; o terceiro seria o PEC+CDS cujo preenchimento e digitação das fichas do CDS nas unidades básicas de saúde e utilização do PEC em alguns ambientes e na recepção (organização da agenda dos profissionais) e por último o PEC onde preenchimento e digitação das fichas do CDS nas unidades básicas de saúde e a utilização do PEC em todos os ambientes da unidade.

Os municípios tiveram que se planejar para implantação e adequação das Unidades de Saúde da Família dependendo do cenário 
Quadro II - Comparação das informações de Documentos gerais e Obrigatórios, endereço, dados clínicos e tratamento contidas no HIPERDIA e formato CDS do e-SUS.

\begin{tabular}{|c|c|}
\hline VARIÁVEIS DO HIPERDIA & VARIÁVEIS DO HIPERDIA NO e-SUS \\
\hline \multicolumn{2}{|r|}{ DOCUMENTOS GERAIS } \\
\hline $\begin{array}{c}\text { Título de eleitor, CTPS, CPF, } \\
\text { PIS/PASEP }\end{array}$ & $\begin{array}{l}\text { Nenhumas dessas informações estão contempladas nas } \\
\text { fichas do formato CDS do e-SUS e apenas o PIS/PASEP } \\
\text { está contemplada na Ficha de Cadastro Individual }\end{array}$ \\
\hline \multicolumn{2}{|r|}{ DOCUMENTOS OBRIGATÓRIOS } \\
\hline Identidade, certidão (TV) & Não contempladas nas fichas cadastrais do e-SUS \\
\hline \multicolumn{2}{|r|}{ ENDEREÇO } \\
\hline $\begin{array}{l}\text { Tipo de logradouro, Nome do } \\
\text { Logradouro, Número, } \\
\text { Complemento, Bairro, CEP, DDD } \\
\text { e Telefone }\end{array}$ & $\begin{array}{c}\text { Todas as estas informações estão contempladas nas } \\
\text { fichas do formato CDS do e-SUS, na Ficha de cadastro } \\
\text { domiciliar }\end{array}$ \\
\hline \multicolumn{2}{|r|}{ DADOS CLÍNICOS } \\
\hline $\begin{array}{l}\text { Pressão Arterial Sistólica, } \\
\text { Pressão Arterial Diastólica, } \\
\text { Cintura, Peso, Altura, Glicemia } \\
\text { Capilar em jejum, Glicemia } \\
\text { capilar Pós Prandial }\end{array}$ & $\begin{array}{l}\text { Essas informações estão contidas A variável Cintura não } \\
\text { está contemplada nas fichas do formato CDS do e-SUS e } \\
\text { todas as demais informações estão contempladas nas } \\
\text { fichas do formato CDS do e-SUS, mas não como valores } \\
\text { mensurados e sim como consolidado de procedimento } \\
\text { apenas. }\end{array}$ \\
\hline \multicolumn{2}{|c|}{ FATORES DE RISCO E DOENÇAS CONCOMITANTES } \\
\hline $\begin{array}{c}\text { Antecedentes Familiares - } \\
\text { cardiovasculares, Diabetes Tipo, } \\
\text { Diabetes Tipo 2, Tabagismo, } \\
\text { Sedentarismo, } \\
\text { Sobrepeso/Obesidade, } \\
\text { Hipertensão Arterial, Infarto } \\
\text { Agudo do Miocárdio, Outras } \\
\text { Coronariopatias, AVC, } \\
\text { Pédiabético, Amputação por } \\
\text { diabetes, Doença Renal }\end{array}$ & $\begin{array}{l}\text { As variáveis - Antecedentes Familiares cardiovasculares, } \\
\text { sedentarismo, Pé Diabético e Amputação por Diabetes } \\
\text { não estão contempladas nas fichas do formato CDS do e- } \\
\text { SUS e todas as demais informações estão incluídas na } \\
\text { Ficha de Cadastro Individual do formato CDS do e-SUS. }\end{array}$ \\
\hline \multicolumn{2}{|r|}{ TRATAMENTO } \\
\hline $\begin{array}{l}\text { Não medicamentoso, } \\
\text { Medicamentoso } \\
\text { (Hidroclorotiaziada, Propranolol, } \\
\text { Captopril, Glibenclamida, } \\
\text { Metformina), comprimidos/dia, } \\
\text { insulina, unidades/dia }\end{array}$ & $\begin{array}{l}\text { Nenhumas dessas informações estão contempladas nas } \\
\text { fichas do formato CDS do e-SUS. }\end{array}$ \\
\hline
\end{tabular}

encontrado, com o aparato tecnológico necessário, conectividade com a rede, recursos humanos necessários para digitação, treinamento de profissionais e segurança das USF's.

Isso fez com que a maioria dos municípios aderisse ao formato CDS e não ao formato PEC, para implantação do e-SUS $A B$, uma vez que no formato CDS o preenchimento das fichas ocorreria nas unidades básicas de saúde e as digitações das fichas poderiam ser feitas em computadores centralizados na Secretaria Municipal de Saúde. O formato PEC, por sua vez, deveria ser implantado e informatizado em alguns ou em todos os ambientes da Unidade Básica de Saúde demandando um maior planejamento e adequação das Unidades.

Acompanhando a maioria dos municípios, João pessoa-PB optou pelo formato CDS, no cenário CDS-SMS, cujo preenchimento das fichas do CDS aconteceria nas unidades básicas de saúde e a digitação das fichas em computadores centralizados nos Distritos Sanitários de Saúde e na Secretaria Municipal de Saúde. 
O Município de João Pessoa optou para o processo de mudança do Sistema de Informação em Saúde da Atenção Básica (SISAB) e enfrentou alguns desafios e incertezas a serem superados. Os coordenadores do sistema de informação em saúde municipal receberam treinamento pelo apoio institucional do MS e em seguida foram realizadas reuniões matriciais por núcleos específicos em cada território do Distrito Sanitário. Além disso, os agentes comunitários de saúde e os profissionais de nível técnico e superior, depois de capacitados dispunham ainda das informações obtidas no site do Departamento de AB e contidas no Manual para preenchimento das Fichas - Sistema com coleta de dados simplificada (CDS).

No inicio, houve um grande número de dúvidas e incertezas que somente puderam ser sanadas durante a prática, necessitando de toda a superação dos desafios da implantação de algo, até então, novo e desconhecido.

Foi observado após reuniões e discussões do grupo condutor, bem como na prática profissional diária, a incorporação de algumas características e desafios anteriormente já identificados no HIPERDIA, bem como novas características oriundas do e-SUS. Pode-se, assim, resumir e apontar as principais características dessa mudança como:

I - Sistemas cada vez mais centralizados e verticalizados onde os dados obedecem ao fluxo sempre na direção do nível local para a central, realizando processamento fundamentalmente no nível central;

II -Ausência de mecanismos de avaliação e controle da qualidade dos dados produzidos uma vez que não ocorre o "feedback" para a Unidade de Saúde da Família (USF);

III - Ênfase na coleta de dados e procedimentos, não permitindo a construção do perfil de saúde da população em nível individual;

IV - Incompatibilidade entre os diversos sistemas de informação utilizados, como também, a indefinição de um sistema ainda em fase de conclusão;

$V$ - Falta/deficiência de infraestrutura de informática, o que dificulta ou até mesmo inviabiliza a coleta adequada e o processamento dos dados, bem como de recursos humanos para digitação das fichas;

Em relação ao fluxo percebe-se a mudança de um sistema para o outro como se pode observálo ao analisar o fluxograma (Figura 2) do percurso do hipertenso nos dois sistemas. No Programa HIPERDIA ${ }^{16}$ o fluxo seguia o percurso onde o ACS durante a visita domiciliar orientava o usuário a procurar a Unidade de Saúda da Família (USF).
O usuário deveria ser acolhido pelos profissionais da USF e, posteriormente, pelo técnico de enfermagem que iria proceder às aferições $e$ mensurações inerentes ao programa HIPERDIA como peso, altura, pressão arterial e circunferência abdominal no sentido de controle da hipertensão. Se o paciente não estivesse com as aferições dentro dos padrões normais e com sintomas de Hipertensão Arterial ou Diabetes Mellitus realizava o monitoramento e após confirmação realização do cadastro no HIPERDIA na USF.

A $1^{\text {a }}$ via do cadastro seria anexada ao prontuário do hipertenso e a $2^{\mathrm{a}}$ via do cadastro seria enviada ao Distrito Sanitário de Saúde que consolidava as informações e enviava a Secretaria Municipal de Saúde, a qual realiza o processamento dos dados para, em seguida, encaminhar para o DATASUS no Ministério da Saúde. A primeira via ficava na própria USF para o acompanhamento do usuário mediante inserção do mesmo em um livro de controle e acompanhamento próprio de cada USF, sempre aprazando (cartão de aprazamento) o retorno de sua consulta. Ao final de cada trimestre a equipe de Saúde da Família deveria se reunir para avaliar o desempenho do Programa HIPERDIA traçando metas para promover melhorias.

Com a chegada do formato CDS do e-SUS, as USF's deixaram de alimentar o sistema do HIPERDIA, não ficando nenhuma via de cadastro, acompanhamento e/ou atendimento na USF, não ocorrendo avaliação, discussão e planejamento de metas e ações para este público. Todo e qualquer usuário ao chegar à USF é acolhido e mediante sua queixa, encaminhado para consulta médica. Este profissional preenche a ficha de atendimento individual que envia para o Distrito Sanitário de Saúde, onde os dados são inseridos no sistema e, em seguida, enviados à Secretaria Municipal de Saúde. A Secretaria Municipal de Saúde irá consolidar a produção de todos os distritos e enviar diretamente para o Departamento da Atenção Básica (DAB) do Ministério da Saúde, e, por conseguinte obtendo um fluxo diferente do HIPERDIA.

Portanto, no formato CDS do e-SUS o usuário só será identificado como hipertenso caso o mesmo durante o cadastro individual realizado pelo ACS ou durante a consulta de um profissional de nível superior, relatar ou apresentar essa condição, uma vez que essa informação só é fornecida pelo curso natural do atendimento, caso contrário não terá essa identificação. No formato do e-SUS CDS as equipes deixaram de realizar a avaliação trimestral de metas e do desenvolvimento das ações e grupos operativos de HIPERDIA. O livro de 
controle e acompanhamento próprio de HIPERDIA da USF foi abolido, ficando apenas como material consultivo em eventuais ações de atendimento e com informações desatualizadas.

O acompanhamento (aprazamento do retorno) e a dificuldade de registro se tornou algo observado com a implantação no formato CDS, o que já tinha sido evidenciado em outro estudo realizado pela coorte de Hipertensão em 2013, cujas dificuldades encontradas pelos autores partiram da ausência dos registros nos prontuários para análise de como estava o acompanhamento dos usuários, fato este potencializado após a utilização do e-SUS formato $\operatorname{CDS}^{17}$

Diferentemente, o HIPERDIA tinha suas ações focadas na situação-problema de saúde referente à Hipertensão Arterial e Diabetes Mellitus onde os usuários eram cadastrados e acompanhados como hipertensos e/ou diabéticos em todas as etapas do cuidado.

Ao considerar o fluxograma da figura 2, se observa que os dados são gerados muito antes de chegar à UBS e que diversas informações são produzidas durante as visitas domiciliares e dentro do próprio consultório, não estando contempladas no preenchimento das fichas seja pelo HIPERDIA seja no formato CDS e-SUS, podendo ser uma problemática advinda da implantação parcial do e-SUS, apenas no formato CDS, estando contempladas ou não no formato PEC e em seus relatórios operacionais suprindo essa deficiência, o que precisa de mais estudos sobre a temática.

Pode-se dizer, então, que as informações que podem alimentar os Sistemas de Informação em Saúde estão sendo aquelas passíveis de quantificação, previamente definidas nestes sistemas, expressas em variáveis numéricas, representando muito mais o trabalho e a produção do profissional, do que a condição de saúde dos usuários, ou mesmo a qualidade do atendimento ofertado pelo profissional e a resolutividade de sua prática em saúde.

Nesse sentido, tanto o HIPERDIA como o e-SUS em seu formato CDS, a exemplo de outros Sistemas de Informação em Saúde, necessitam ser repensados, no sentido de incluir outras formas de informação produzidas pelos ACS's e pelos demais profissionais da área da saúde ${ }^{18}$.

Ao analisar os relatórios produzidos pelos sistemas percebe-se que no SIS/HIPERDIA, os dados produzidos na UBS eram referentes aos usuários hipertensos acompanhados mensalmente, sendo que esses registros demonstravam o número de consulta e controle de dispensa das medicações padronizadas pelo Ministério da Saúde ${ }^{19}$. Enquanto que os dados provenientes do e-SUS no formato CDS são gerados durante as consultas, cadastros, procedimentos, visitas domiciliar e atividades coletivas não referentes a usuários hipertensos, mas usuários como um todo em suas diversas patologias.

É importante frisar que embora os dados tenham uma fonte comum, não se cruzam para a produção de informações sobre a resolutividade da atenção à saúde aos usuários hipertensos atendidos na UBS.

Outro problema que surge com a divergência de produção, fluxo e análise dos dados entre os dois SISs é em relação à aquisição e dispensa de medicamentos para a Atenção Básica, sendo que este é, justamente, um dos principais objetivos do SIS/HIPERDIA ${ }^{20}$. O e-SUS no formato CDS não gera este tipo de informação relacionado ao tratamento do Hipertenso e Diabético, o planejamento das ações assistenciais e da dispensa de medicamentos que estava relacionado ao número de usuários cadastrados na $U_{B S}^{20,21}$ ficando interligado a dispensação na própria farmácia da UBS e seu consolidado final de medicamentos.

O modo como se configura o fluxo da informação aqui analisado, diverge da proposta do MS que visa disponibilizar os dados do e-SUS para fornecer informações que embasem a tomada de decisão pelos gestores do SUS, bem como, possibilitar a instrumentalização das instâncias de Controle Social disponibilizando, assim, os dados para o uso de todos os envolvidos na consolidação do SUS 22 .

Essa afirmação é reforçada pelo fato de que os profissionais da APS não possuem acesso ao sistema nem aos relatórios gerados pelo sistema e-SUS no formato CDS, podendo esta instância observar apenas os dados já condensados nos relatórios mensais por USF.

Entretanto, com o advento do formato CDS do e-SUS acaba a fragmentação do indivíduo em vários sistemas onde, por exemplo, uma única mulher poderia anteriormente entrar no SIS/PréNatal, no SIS/Colo, no SIS/HIPERDIA e no SIAB.

Neste caso, o ideal seria um modelo de Sistema de Informação em Saúde no qual todas as informações pudessem ser submetidas e modeladas ao que está pré-estabelecido. O que se percebe é que os dados se restringem a quantificar consultas, procedimentos, exames e visitas domiciliares realizadas, dentre outros itens de igual natureza.

\section{CONSIDERAÇÕES FINAIS}

O presente estudo possibilitou a discussão 
dos dados referentes à hipertensão arterial a partir de duas bases investigadas, contidas no HIPERDIA e do formato CDS do e-SUS. As dificuldades no cruzamento de dados e informações dessas duas bases de dados demonstram as distorções existentes a respeito de dados que deveriam estar em consonância. Assim, a contribuição deste estudo está em mostrar a necessidade de se pensar novas possibilidades na geração da informação em saúde dos hipertensos no Brasil.

O empoderamento na utilização dos sistemas de informação por profissionais de saúde faz-se necessário pela facilidade que esse tipo de instrumento gera na visualização do quadro de saúde de populações específicas, como é o caso das pessoas que vivem com HAS. Sabe-se, no entanto, que o sucesso na utilização desses instrumentos não depende exclusivamente da vontade dos profissionais em utilizá-lo, mas também de estrutura informacional que seja capaz de sustentar o seu uso nos níveis locais de planejamento e assistência à saúde e de capacidade técnica necessária para transformar os dados em informação útil ao planejamento.

Figura 2 - Fluxograma Analisador do percurso do usuário até geração da informação de hipertensão arterial e envio de dados com o HIPERDIA (sem preenchimento) e formato CDS do e-SUS (com preenchimento)

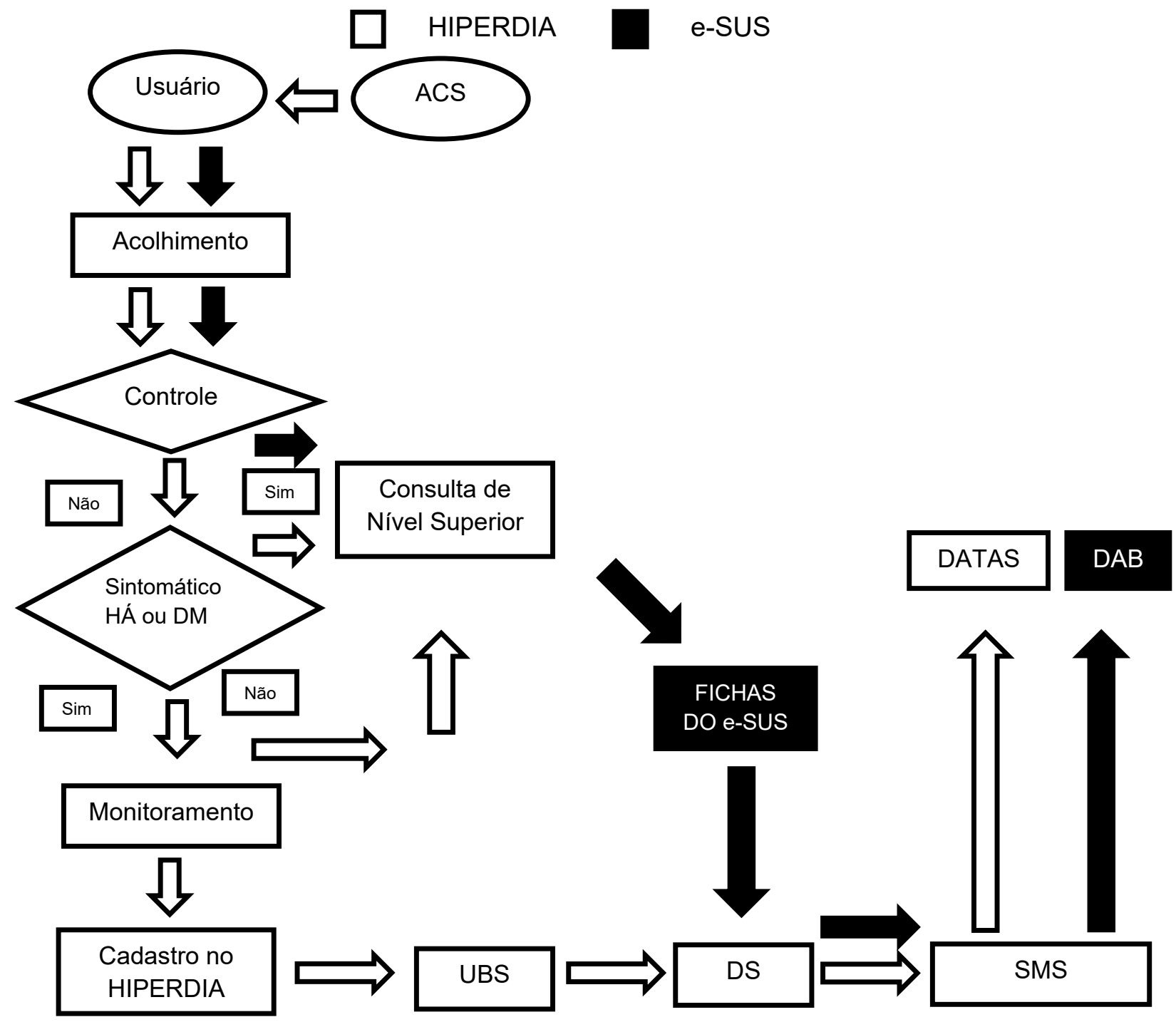

Fonte: Elaboração própria 
Este estudo aponta sua limitação referente à literatura que discuta e reflita sobre a produção, fluxo e análise dos dados do e-SUS, visto ser ainda escassa na literatura científica face ao curto período decorrente de sua implantação.

Portanto, se fazem necessárias a realização de novas pesquisas, estudos e relatos em longo prazo, para que realmente se possa melhor avaliar o e-SUS, no sentido de analisá-lo enquanto ferramenta de processo de planejamento,

\section{REFERÊNCIAS}

1. Cipullo JP, Martin JFV, Ciorlia LAS, de Godoy MRP, Cação JC, Loureiro AAC, Cesarino CB, Carvalho AC, Cordeiro JA, Burdmann EA. Prevalência e Fatores de Risco para Hipertensão em uma População Urbana Brasileira. Arquivos Brasileiros de Cardiologia. 2009, 94(4):519-526.

2. Malfatti CRM, Assunção AN. Hipertensão arterial e diabetes na Estratégia de Saúde da Família: uma análise da frequência de acompanhamento pelas equipes de Saúde da Família. Ciência \& Saúde Coletiva. 2011; 16(1):1383-1388.

3. Sociedade Brasileira de Cardiologia / Sociedade Brasileira de Hipertensão / Sociedade Brasileira de Nefrologia. VI Diretrizes Brasileiras de Hipertensão. Arquivos Brasileiros de Cardiologia. 2010; 95(supl.1):1-51.

4. Neves EB. Prevalência de sobrepeso e obesidade em militares do exército brasileiro: associação com a hipertensão arterial. Ciênc Saúde Coletiva [serial on the internet]. 2008 [cited 2015 Jun 16];13(5):1661-8. Available from: <http://www.scielo.br/ $\mathrm{pdf} / \mathrm{csc} / \mathrm{v} 13 \mathrm{n} 5 / 29 . \mathrm{pdf}>$

5. Castro ME, Rolim MO, Mauricio TF. Prevenção da hipertensão e sua relação com o estilo de vida de trabalhadores. Acta Paul. Enferm [serial on the internet]. 2005 [cited 2015 Jun 16];18(2):184-9. Available from: http://www.scielo.br/pdf/ape/ v18n2/a11v18n2.pdf

6. Passos VMA, Assis TD, Barreto SM. Hipertensão arterial no Brasil: estimativa de prevalência a partir de estudos de base populacional. Epidemiologia e Serviços de Saúde. 2006; 15(1):35-45

7. BRASIL. Plano de ações estratégicas para o enfrentamento das doenças crônicas não transmissíveis (DCNT) no Brasil 2011-2022. Brasília: Ministério da Saúde, 2011

8. Brasil. Ministério da Saúde. Secretaria de Atenção à Saúde. Departamento de Atenção Básica. Estratégias para o cuidado da pessoa com doença crônica: hipertensão arterial sistêmica / Ministério da Saúde, Secretaria de Atenção à Saúde, Brasília, 2013.

9. Chazan AC, Perez EA. Avaliação da implementação do sistema informatizado de cadastramento e acompanhamento de hipertensos e diabéticos (HIPERDIA) nos municípios do estado do Rio de Janeiro. Rev APS. 2008; jan/mar; 11(1):10-6.

10. Ministério da Saúde (Brasil). Portaria GM/MS no 1412, de 10 de julho de 2013. Institui o Sistema de Informação em Saúde para a Atenção Básica (SISAB). Diário Oficial da União 11 jul 2013;Seção 1.

11. Paes NA. Avaliação da efetividade do controle da hipertensão arterial sistêmica e associação com os fatores de risco comparando a atenção do Programa de Saúde da Família e de Unidades Básicas de Saúde de municípios do nordeste do Brasil. Projeto CNPq. Edital: MCT/CNPq/MS - SCTIE DECIT/MS No. 37/2008. Tema: G. DOENÇAS DO APARELHO CIRCULATÓRIO, 2008.

12. Paes NA. Desempenho do Programa de Saúde da Família comparado com o das Unidades Básicas de Saúde no controle da Hipertensão Arterial Sistêmica e fatores execução, monitoramento e avaliação das ações em saúde e dos profissionais e de cuidado, bem como a sua implantação em formato completo (CDS+PEC). Apesar de assentar em pressupostos importantes, como a possibilidade de superar a fragmentação das informações, o e-SUS formato CDS ainda é verticalizado e centralizado, onde se cometem desta forma, os mesmos erros de outros SIS.

associados em Municípios do Estado da Paraíba: Um estudo de coorte longitudinal. Projeto CNPq. Edital MCT/CNPq N. ${ }^{\circ}$ 67/2009, 2009a.

13. Paes NA. Desempenho do Programa de Saúde da Família comparado com o das Unidades Básicas de Saúde no controle da Hipertensão Arterial Sistêmica e fatores associados em Municípios do Estado da Paraíba: Um estudo de coorte longitudinal. Projeto FAPESC. Edital FAPESC N. 02/2009, 2009b.

14. Ministério da Saúde (Brasil). Portaria MS/GM no 2.488 , de 21 de outubro de 2011. Redefine os parâmetros de vinculação dos Núcleos de Apoio à Saúde da Família (NASF) modalidades 1 e 2 às equipes de Saúde da Família e/ou Atenção Básica para populações específicas, cria a modalidade NASF 2, e dá outras providências. Diário Oficial da União 31 dez 2012;Seção 1.

15. Ministério da Saúde (Brasil). e-SUS Atenção Básica: Manual do Sistema com Coleta de Dados Simplificada: CDS - Versão 2.0 [recurso eletrônico]. Secretaria-Executiva. Brasília Ministério da Saúde, 2015.

16. Ministério da Saúde (Brasil). Sistema de Cadastramento e Acompanhamento de Hipertensos e Diabéticos - HIPERDIA. 2008. [Citado em 2015 jun 19]. Disponível em: http://hiperdia. datasus.gov.br.

17. Araújo IM, Paes NA. Qualidade dos dados antropométricos dos usuários hipertensos atendidos no programa de Saúde da Família e sua associação com fatores de risco. Texto Contexto Enferm, Florianópolis, 2013 Out-Dez; 22(4): 1030-40.

18. Moraes IHS, Santos SRFR. Informações para gestão do SUS: necessidades e perspectivas. Informe epidemiológico do SUS. 2001 Jan-Mar; 10(1):49-56.

19. Ministério da Saúde (Brasil). Secretaria de Atenção à Saúde Departamento de Atenção Básica. SIAB - manual do sistema de informação da atenção básica. Brasília (DF): MS; 2003. 7. Ministério da Saúde (BR)

20. Brasil. Ministério da Saúde. Secretaria de Políticas Públicas Plano de Reorganização da Atenção à Hipertensão Arteria e Diabetes Mellitus. Rev Saúde Púb. 2001 Dez; 35(6):585-8.

21. Brasil. Ministério da Saúde. Portaria ${ }^{\circ} 371 / \mathrm{GM}$ de 4 de março de 2002. Dispõe sobre o Programa Nacional de Assistência Farmacêutica para Hipertensão Arterial e Diabetes mellitus. Brasília: Diário Oficial da União de 6 de março de 2002, Seção 1, página 88. [acesso 2015 Jun 13]. Disponível em: http:// hiperdia.datasus.gov. br/manuais/portaria ministerial371.zip

22. Moraes IHS, Santos SRFR. Informações para gestão do SUS: necessidades e perspectivas. Informe epidemiológico do SUS. 2001 Jan-Mar; 10(1):49-56.15.

\section{CORRESPONDÊNCIA}

Jairo Domingos de Morais

Rua Fernando Luíz Henrique dos Santos, 451, Apto 104,

Bessa, João Pessoa, CEP: 58037-50

E-mail: jairodmfisio@hotmail.com 